\title{
Exercise Capacity in Prepubertal Children with Cystic Fibrosis
}

\author{
Emma Kilbride, ${ }^{1,2}$ John Widger, ${ }^{1}$ Juliette Hussey, ${ }^{3}$ Basil El Nazir, ${ }^{1}$ and Peter Greally \\ ${ }^{1}$ The Adelaide and Meath Hospital, Incorporating The National Children's Hospital, Dublin 24, Ireland \\ ${ }^{2}$ Children's Respiratory Lab, The National Children's Hospital, Tallaght, Dublin 24, Ireland \\ ${ }^{3}$ Discipline of Physiotherapy, School of Medicine, Trinity College Dublin, Dublin 2, Ireland \\ Correspondence should be addressed to Emma Kilbride, emma.kilbride@amnch.ie \\ Received 28 February 2012; Accepted 2 May 2012 \\ Academic Editors: A. Celi and A. Yokoyama
}

Copyright (c) 2012 Emma Kilbride et al. This is an open access article distributed under the Creative Commons Attribution License, which permits unrestricted use, distribution, and reproduction in any medium, provided the original work is properly cited.

Background. Patients with cystic fibrosis (CF) are observed to have diminished lung function, nutritional status, and aerobic exercise capacity. All three parameters are related to prognosis and survival. However, there is little information regarding these parameters in prepubertal patients. Methods. Our study groups consisted of sixteen patients with CF (7 girls) and 99 healthy volunteers (52 girls). Subjects performed spirometry and a progressive exercise test to exhaustion on a cycle ergometer. Leg muscle strength was measured using an isokinetic dynamometer. Physical activity was examined using the modifiable activity questionnaire and accelerometer. Results. Nutritional status was similar between groups (BMI-boys control versus CF 18.5 versus 17.9, girls control versus CF 19.5 versus 17.4). Girls with CF were significantly smaller and lighter than controls. Lung function was significantly reduced in $\mathrm{CF}$ groups $\left(\mathrm{FEV}_{1}\right.$ - boys control versus $\mathrm{CF} 91 \%$ versus $84 \%$, girls control versus $\mathrm{CF} 90 \%$ versus $82 \%$ ). Patients with $\mathrm{CF}$ were as active and as fit as their healthy controls. Conclusion. In this group of prepubertal children with CF, nutritional status was comparable to healthy children of the same age. Their aerobic exercise tolerance and peripheral muscle strength were also relatively well preserved despite significantly lower lung function.

\section{Introduction}

Cystic Fibrosis, an autosomal recessive disorder, is the most common lethal genetic disease affecting Caucasians [1]. Patients with CF are observed to have diminished lung function, nutritional status, and aerobic exercise capacity. All three parameters are related to overall survival. Indeed a higher $\mathrm{VO}_{2} \mathrm{max}$ has been reported to be a marker for longer survival in both children and adults with CF $[2,3]$. In healthy children, maximum physical performance is related to both body mass (body weight and fat free mass) and cardiopulmonary capacity. It has been suggested that, in children with $\mathrm{CF}$, poor nutritional status and decreased muscle mass may lead to exercise limitation and also that pulmonary function is significantly related to exercise tolerance when $\mathrm{FEV}_{1}$ is significantly diminished $[4,5]$. In addition to deterioration in lung function, children with CF have been shown to have lower muscle strength and physical activity (PA) levels when compared to healthy control subjects [6-8].
To date, the information regarding these parameters specific to prepubertal children with CF is lacking. We hypothesised that prepubertal children with CF would have lower aerobic exercise capacity, peripheral muscle strength and reduced PA when compared to healthy, age-matched controls.

\section{Materials and Methods}

2.1. Subjects. Our subjects were recruited from a list of patients regularly attending the Cystic Fibrosis Clinic of the National Children's Hospital in Dublin. In total, 24 patients (14 boys, 10 girls) were in the desired age range of 1012 years. Four patients were excluded due to MRSA, and a further four were excluded, as they were unable to perform a reliable exercise test. Our final study group consisted of 16 patients with CF ( 7 girls). Our control group consisted of 99 healthy volunteers (52 girls). They were recruited for another 
study and came from randomly selected primary schools in the Dublin area. No subjects were taking oral steroids at the time of testing. Genotype of the CF group consisted of $n=6$ homozygous for $\Delta \mathrm{F} 508$ and $n=10$ heterozygous for $\Delta \mathrm{F} 508$. Twelve children were pancreatic insufficient. Approval for the study was obtained from the Research Ethics Committee of SJH/AMNCH.

2.2. Anthropometry. Height was measured using a wallmounted stadiometer (Holtain Ltd., UK). Weight was measured using an electronic scale (Seca Corporation, CA, USA). Subjects were minimally clothed and barefoot for both measurements. Lean body mass was measured by bioelectrical impedance analysis using a Tanita lean body mass scales (Tanita Corporation, Tokyo, Japan).

2.3. Lung Function and Cardiopulmonary Exercise Testing. Spirometry was performed prior to exercise using the spirometry module of the Vmax Encore exercise system (VIASYS Healthcare). The exercise test was performed on a cycle ergometer (Ergoselect 200P, Ergoline, Germany). Once the subject was seated comfortably on the ergometer, a 3-lead ECG (Sensormedics) and a pulse oximeter (Nonin 8600, USA) with finger probe were attached. For the duration of the test, subjects wore a nose clip and breathed through a mouthpiece connected to a mass flow sensor. This allowed respiratory variables to be measured and recorded on a breath by breath basis. All equipment and systems were calibrated prior to testing according to specific protocols. Raw data from each test was imported into a Microsoft Excel worksheet. Resting, warmup, and $\mathrm{VO}_{2}$ max values were calculated by taking a 20 -second average at the end of the appropriate resting or exercise period.

The exercise test began with a 2 -minute rest period. Subjects then began to cycle at an initial load of 20 watts for a 1-minute warm-up period, followed by a continuous ramp increase in workload of 15 or 20 watts/minute. Subjects exercised for as long as possible against the ever-increasing workload. Once the subject reached exhaustion, the workload was reduced to the warm-up level of 20 watts for a further 1-minute recovery period. During exercise, subjects were asked to maintain a constant pedalling speed of between 60 and $80 \mathrm{rpm}$ and this was displayed in digital format in front them. All subjects were verbally encouraged to continue cycling for as long as possible.

2.4. Muscle Strength. Leg muscle strength was measured using the Biodex III Isokinetic Dynamometer (Biodex Medical, USA). The knee flexors and extensors of the dominant leg were tested at three speeds of movement $\left(60^{\circ} / \mathrm{sec}, 90^{\circ} / \mathrm{sec}\right.$, and $180^{\circ} / \mathrm{sec}$ ). Children were seated for the test with belts attached around the chest, waist, and leg to isolate the muscle group being tested. The test required the child to kick out against the dynamometer arm from a position of $90^{\circ}$ flexion to full extension and to immediately pull back to the starting position. Five repetitions were performed at speeds of $60^{\circ} / \mathrm{sec}$ and $90^{\circ} / \mathrm{sec}$ and thirty repetitions at $180^{\circ} / \mathrm{sec}$. There was a 30 -second rest period between each set of repetitions.
Isokinetic testing permits examination of muscle strength throughout a range of movement. It is accepted as a valid and reproducible method of assessing muscle strength and is considered the gold standard in orthopaedics and sports medicine.

2.5. Physical Activity. Physical activity was measured both subjectively, using a modification of the modifiable activity questionnaire (MAQ) [9] and objectively using a Triaxial Accelerometer (RT3 research tracker, Stayhealthy). The MAQ required children, along with their parents, to list all organised activities they participated in on a regular basis, noting months per year, days per week, and minutes per day spent at each activity. Results were summed and expressed as an average of total hours of activity per day. The relative intensity of each activity was also estimated by multiplying the hours per day by the metabolic cost (MET) of that activity [10], and results were expressed as total MET-h/day. The RT3 tracker measures acceleration in three individual planes and integrates it into one value, the vector magnitude. The accelerometer was worn for three consecutive days. Results were analysed according to the number of activity counts per minute as follows; time spent inactive $(0-99 \mathrm{cpm})$, light activity $(100-970 \mathrm{cpm})$, moderate activity $(971-2333 \mathrm{cpm})$, and vigorous activity $(>2334 \mathrm{cpm})[11]$. Results were averaged over the three days and expressed as percentage of total time worn.

2.6. Statistics. Statistics were performed using MINITAB 14. Data was tested for normality using an Anderson-Darling test. An unpaired $t$-test was used for comparison between normally distributed data. Significance for all tests was set at $P<0.05$.

\section{Results}

Table 1 shows anthropometry and lung function results for controls and children with CF. Within both groups, boys and girls were of similar age, height, weight, and body mass index (BMI). Boys with CF were of similar age, height, weight, BMI, and LBM compared to control boys. Girls with CF were significantly shorter and lighter than control girls; however, LBM and BMI values were not significantly different. Lung function $\left(\mathrm{FEV}_{1}\right)$ was significantly reduced in both $\mathrm{CF}$ groups compared to their controls. $\mathrm{FEV}_{1}$ ranged from 64 to $91 \%$ predicted in girls with $\mathrm{CF}$ and from 72 to $97 \%$ predicted in boys with CF. In relation to our CF centre as a whole, we have 66 patients capable of performing lung function. We found no significant difference in mean $\mathrm{FEV}_{1}$ in our CF study group compared to our CF centre as a whole (mean $\mathrm{FEV}_{1} \pm \mathrm{SEM}$, CF study group: $83 \% \pm 2.4$ versus CF centre: $80 \% \pm 2.7$, $P=0.62$ ).

All subjects completed the progressive exercise test to exhaustion. Mean heart rate (HR) and respiratory quotient (RQ) in all groups were greater than $185 \mathrm{bpm}$ and 1.10 , respectively. Each subject also showed physical signs of exhaustion towards the end of the test, indicating a maximal effort was achieved. Results for exercise and muscle strength 
TABLE 1: Anthropometry and lung function.

\begin{tabular}{lcccc}
\hline \multirow{2}{*}{ Mean (SEM) } & \multicolumn{2}{c}{ Control group } & \multicolumn{2}{c}{ CF group } \\
& Boys $(n=47)$ & Girls $(n=52)$ & Boys $(n=9)$ & $11.3(0.3)$ \\
Age & $10.9(0.1)$ & $11.2(0.1)$ & $143(1.3)$ & $10.7(0.3)$ \\
Height (cm) & $146(1.0)$ & $147(1.0)$ & $36.4(1.2)$ & $138^{\phi}(5.3)$ \\
Weight (kg) & $39.8(1.2)$ & $42.5(1.3)$ & $17.9(0.5)$ & $33.8^{\phi}(4.5)$ \\
BMI & $18.5(0.4)$ & $19.5(0.5)$ & $80(1.3)$ & $17.4(1.5)$ \\
\% LBM & $78(1.2)$ & $75^{*}(0.8)$ & $91(3.4)$ & $82(3.8)$ \\
FVC (\%predicted) & $94(1.7)$ & $94(1.4)$ & $84^{\phi}(2.7)$ & $84^{\phi}(3.9)$ \\
FEV $_{1}$ (\%predicted) & $92(1.1)$ & $90(1.2)$ & $74^{\phi}(5.5)$ & $86(10.6)$ \\
FEF $_{25-75 \%}$ (\%predicted) & $92(0.2)$ & $97(0.0)$ & \\
\hline
\end{tabular}

Mean (SEM) age, height, weight, body mass index; BMI, lean body mass; LBM, forced vital capacity; FVC, forced expiratory volume in one second; FEV forced expiratory flow; $\mathrm{FEF}_{25-75 \%}$. ${ }^{*}$ Indicates a significant gender difference within group, $\phi$ indicates a significant difference from corresponding control group. $P<0.05$.

tests are shown in Table 2. While $\mathrm{VO}_{2} \max$ was significantly lower in control girls compared to control boys, there was no such gender difference in the CF group. Boys and girls with $\mathrm{CF}$ were as fit as their healthy controls with no significant differences in $\mathrm{VO}_{2} \max$. Muscle strength was significantly lower in control girls compared to control boys at the highest speed of movement $\left(\mathrm{E} 180^{\circ} / \mathrm{sec}\right.$ and $\left.\mathrm{F} 180^{\circ} / \mathrm{sec}\right)$. There was no significant difference in muscle strength between control and CF groups.

The modifiable activity questionnaire (MAQ) showed control boys to be significantly more active than control girls (Table 3). Boys and girls with CF were equally active and were also as active as their corresponding controls.

\section{Discussion}

This study found that, compared to healthy age-matched controls, aerobic fitness, muscle strength, and physical activity (PA) levels in this group of children with CF were relatively well preserved despite a significant deterioration in lung function.

Mean $\mathrm{VO}_{2}$ max in our control group was slightly higher than previous studies examining exercise capacity in healthy children of this age [12-14], indicating a relatively fit group of children. In keeping with the literature to date, a significant gender difference in $\mathrm{VO}_{2}$ max was seen in the control group, with higher fitness levels in boys compared to girls. Several studies have examined $\mathrm{VO}_{2}$ max in children with CF; however, the age range tends to be large (7-18 years). Relatively few studies have looked at $\mathrm{VO}_{2}$ max specifically in prepubertal children with CF. Stanghelle et al. [15] reported a mean $\mathrm{VO}_{2} \max$ of $48.6 \mathrm{~mL} / \mathrm{kg} / \mathrm{min}$ in a group of $10 \mathrm{prepu}-$ bertal children with CF. While more recently Selvadurai et al. [16] reported a mean $\mathrm{VO}_{2}$ max value of $41.7 \mathrm{~mL} / \mathrm{kg} / \mathrm{min}$ in a similar but larger group $(n=70)$. The fitness levels of our CF group lay in between these two studies at $44.9 \mathrm{~mL} / \mathrm{kg} / \mathrm{min}$ with no significant difference between boys and girls.

In comparison to healthy control subjects, aerobic fitness of our boys and girls with CF was not significantly different. Previous studies comparing the exercise capacity of children with $\mathrm{CF}$ to healthy controls have found $\mathrm{VO}_{2} \max$ to be significantly lower in CF even when corrected for body weight $[6,17]$. However, in these studies, BMI was significantly reduced in CF groups suggesting poor nutritional status. In our study, boys and girls with CF had a similar nutritional status (BMP and LBM) when compared to their healthy controls. This favourable nutritional status could help to account for the similar $\mathrm{VO}_{2}$ max values reported here.

While aerobic exercise capacity compared favourably between CF groups and controls, lung function did not. It has been shown previously that, in adults with CF, measurements of lung function correlate poorly with exercise capacity as measured by $\mathrm{VO}_{2} \max$ [18]. In children with CF, de Meers et al. [4] reported a significant correlation between lung function and $\mathrm{VO}_{2}$ max when $\mathrm{FEV}_{1}$ was $<80 \%$ predicted. However, Cropp et al. [5] reported a significant correlation only when lung function was $<60 \%$ predicted. In this study, $\mathrm{FEV}_{1}$ ranged from 72 to $97 \%$ predicted in boys with CF and $64-91 \%$ predicted in girls with CF, and we found no relationship between lung function and $\mathrm{VO}_{2}$ max in either $\mathrm{CF}$ group.

Physical activity is an important factor in the growth and development of children, and maintaining high levels of PA is especially important in the management of CF. Studies have shown that, in children with $\mathrm{CF}$, those with more active lifestyles have significantly greater aerobic capacity, quality of life and nutrition, and significantly lower disease severity than children with lower activity levels $[16,19,20]$. Relatively few studies have compared PA levels in children with CF to healthy controls. Selvadurai et al. [16] and Nixon et al. [6] both reported no significant differences in PA levels, but the latter reported patients with CF spending significantly less time at vigorous activities. In the present study, when reported both subjectively by questionnaire and objectively by accelerometer, patients with CF were shown to be as active as their healthy controls, in terms of both overall activity levels and time spent at vigorous activities. The finding that our patients with CF were as active as our controls, even when lung function was so significantly reduced, may help to explain their well-maintained aerobic capacity.

Muscle strength was an important factor to consider when comparing exercise tolerance between our two groups. 
TABLE 2: Exercise and muscle strength.

\begin{tabular}{|c|c|c|c|c|}
\hline \multirow{2}{*}{ Mean (SEM) } & \multicolumn{2}{|c|}{ Control group } & \multicolumn{2}{|c|}{ CF group } \\
\hline & Boys $(n=47)$ & Girls $(n=52)$ & Boys $(n=9)$ & Girls $(n=7)$ \\
\hline $\mathrm{VO}_{2} \max (\mathrm{ml} / \mathrm{kg} / \mathrm{min})$ & $48.5(1.4)$ & $40.6^{*}(1.0)$ & $46.7(2.1)$ & $41.9(3.3)$ \\
\hline WLmax (watts) & $112(2.7)$ & $100 *(2.8)$ & $104(6.8)$ & $89(6.8)$ \\
\hline $\mathrm{E} 60^{\circ} / \mathrm{sec}$ & $158(4.5)$ & $159(6.4)$ & $147(11.2)$ & $151(14.0)$ \\
\hline $\mathrm{E} 90^{\circ} / \mathrm{sec}$ & $146(4.1)$ & $138(5.5)$ & $142(15.5)$ & $142(15.5)$ \\
\hline $\mathrm{E} 180^{\circ} / \mathrm{sec}$ & $116(2.6)$ & $104^{*}(3.7)$ & $122(12.7)$ & $107(6.9)$ \\
\hline $\mathrm{F} 60^{\circ} / \mathrm{sec}$ & $76(3.4)$ & $73(4.0)$ & $72(7.2)$ & $60(9.2)$ \\
\hline $\mathrm{F} 90^{\circ} / \mathrm{sec}$ & $76(3.7)$ & $67(3.0)$ & $80(7.4)$ & $71(10.8)$ \\
\hline $\mathrm{F} 180^{\circ} / \mathrm{sec}$ & $74(2.6)$ & $58^{*}(2.2)$ & $77(4.9)$ & $65(6.7)$ \\
\hline
\end{tabular}

Mean (SEM) maximum oxygen consumption; $\mathrm{VO}_{2} \max$, maximum workload; WLmax, mean muscle strength: extension; $\mathrm{E}$ and flexion; $\mathrm{F}$ at 60 , 90 , and $180^{\circ} /$ sec. ${ }^{*}$ Indicates a significant gender difference within group. $P<0.05$.

TABLe 3: Physical activity data.

\begin{tabular}{lcccc}
\hline Mean (SEM) & \multicolumn{2}{c}{ Control group } & \multicolumn{2}{c}{ CF group } \\
\hline PA (h/day) & Boys & Girls & $0.8(0.2)$ & $0.6(0.2)$ \\
PA (MET h/day) & $1.0(0.1)$ & $0.8^{*}(0.1)$ & $5.5(1.1)$ & $3.1(0.9)$ \\
Light (\%) & $7.3(0.6)$ & $4.4^{*}(0.4)$ & $42(4.7)$ & $49(4.0)$ \\
Moderate (\%) & $43(2.6)$ & $45(2.0)$ & $11(1.6)$ & $13(2.5)$ \\
Hard/vig (\%) & $16(4.2)$ & $12(1.6)$ & $4(1.2)$ & $3(0.9)$ \\
\hline
\end{tabular}

Mean (SEM) hours per day physical activity; PA, metabolic equivalent for physical activity; MET PA measured by MAQ. Percentage of time spent at light, moderate, and hard/vigorous physical activity as measured by accelerometer. ${ }^{*}$ Indicates a significant gender difference within group. $P<0.05$.

Previous studies looking at muscle strength in children with CF are limited. Selvadurai et al. [8], using a similar protocol to ours, examined muscle strength in 13 -year-old boys with $\mathrm{CF}$ and found a significant reduction in leg muscle strength compared to healthy controls. Similarly, Selvadurai et al. [8] examined muscle strength in young female athletes with CF aged 14 years. Again they found significantly lower values in $\mathrm{CF}$ versus healthy controls despite similar $\mathrm{VO}_{2}$ max values. In our study, muscle strength was similar between children with CF and their healthy controls at all levels of resistance. Perhaps the young age of our study group, their relatively good lung function, along with their high PA levels could help explain their well-maintained muscle strength. One of the limitations recognised in this study is the limited sample size, however, in a specific cohort such as this recruiting large numbers can be difficult.

In conclusion, we found in a small group of prepubertal children with CF that nutritional status and physical activity levels were comparable to healthy children of the same age. Aerobic exercise capacity and muscle strength were also relatively well preserved despite a significant reduction in lung function.

\section{Disclosure}

Data presented at the 22nd Annual North American Cystic Fibrosis Conference, Orlando, Florida, 23rd-25th October 2008 and the Irish Thoracic Society annual scientific meeting, Dublin, Ireland, 8th-10th November 2007.

\section{References}

[1] B. J. Rosenstein and P. L. Zeitlin, "Cystic fibrosis," The Lancet, vol. 351, no. 9098, pp. 277-282, 1998.

[2] P. A. Nixon, D. M. Orenstein, S. F. Kelsey, and C. F. Doershuk, "The prognostic value of exercise testing in patients with cystic fibrosis," The New England Journal of Medicine, vol. 327, no. 25, pp. 1785-1788, 1992.

[3] P. Pianosi, J. LeBlanc, and A. Almudevar, "Peak oxygen uptake and mortality in children with cystic fibrosis," Thorax, vol. 60, no. 1, pp. 50-54, 2005.

[4] K. De Meer, V. A. M. Gulmans, and J. Van Der Laag, "Peripheral muscle weakness and exercise capacity in children with cystic fibrosis," American Journal of Respiratory and Critical Care Medicine, vol. 159, no. 3, pp. 748-754, 1999.

[5] G. J. Cropp, P. Pullano, F. J. Cerny, and I. T. Nathanson, "Exercise tolerance and cardiorespiratory adjustments at peak work capacity in cystic fibrosis," American Review of Respiratory Disease, vol. 126, no. 2, pp. 211-216, 1982.

[6] P. A. Nixon, D. M. Orenstein, and S. F. Kelsey, "Habitual physical activity in children and adolescents with cystic fibrosis," Medicine and Science in Sports and Exercise, vol. 33, no. 1, pp. 30-35, 2001.

[7] J. Hussey, J. Gormley, G. Leen, and P. Greally, "Peripheral muscle strength in young males with cystic fibrosis," Journal of Cystic Fibrosis, vol. 1, no. 3, pp. 116-121, 2002.

[8] H. C. Selvadurai, J. Allen, T. Sachinwalla, J. Macauley, C. J. Blimkie, and P. P. Van Asperen, "Muscle function and resting energy expenditure in female athletes with cystic fibrosis," American Journal of Respiratory and Critical Care Medicine, vol. 168, no. 12, pp. 1476-1480, 2003. 
[9] D. J. Aaron, A. M. Kriska, S. R. Dearwater, J. A. Cauley, K. F. Metz, and R. E. LaPorte, "Reproducibility and validity of an epidemiologic questionnaire to assess past year physical activity in adolescents," American Journal of Epidemiology, vol. 142, no. 2, pp. 191-201, 1995.

[10] B. E. Ainsworth, W. L. Haskell, M. C. Whitt et al., "Compendium of physical activities: an update of activity codes and MET intensities," Medicine and Science in Sports and Exercise, vol. 32, no. 9, pp. S498-S504, 2000.

[11] J. Hussey, C. Bell, K. Bennett, J. O’Dwyer, and J. Gormley, "Relationship between the intensity of physical activity, inactivity, cardiorespiratory fitness and body composition in 7-10year-old Dublin children," British Journal of Sports Medicine, vol. 41, no. 5, pp. 311-316, 2007.

[12] A. Vinet, S. Mandigout, S. Nottin et al., "Influence of body composition, hemoglobin concentration, and cardiac size and function of gender differences in maximal oxygen uptake in prepubertal children," Chest, vol. 124, no. 4, pp. 1494-1499, 2003.

[13] M. Dencker, O. Thorsson, M. K. Karlsson et al., "Gender differences and determinants of aerobic fitness in children aged 8-11 years," European Journal of Applied Physiology, vol. 99, no. 1, pp. 19-26, 2007.

[14] N. S. Rizzo, J. R. Ruiz, A. Hurtig-Wennlof, F. B. Ortega, and M. Sjostrom, "Relationship of physical activity, fitness, and fatness with clustered metabolic risk in children and adolescents: the European Youth Heart Study," Journal of Pediatrics, vol. 150, no. 4, pp. 388-394, 2007.

[15] J. K. Stanghelle, N. Hjeltnes, H. Michalsen, H. J. Bangstad, and D. Skyberg, "Pulmonary function and oxygen uptake during exercise in 11-year-old patients with cystic fibrosis," Acta Paediatrica Scandinavica, vol. 75, no. 4, pp. 657-661, 1986.

[16] H. C. Selvadurai, C. J. Blimkie, P. J. Cooper, C. M. Mellis, and P. P. Van Asperen, "Gender differences in habitual activity in children with cystic fibrosis," Archives of Disease in Childhood, vol. 89, no. 10, pp. 928-933, 2004.

[17] C. Moser, P. Tirakitsoontorn, E. Nussbaum, R. Newcomb, and D. M. Cooper, "Muscle size and cardiorespiratory response to exercise in cystic fibrosis," American Journal of Respiratory and Critical Care Medicine, vol. 162, no. 5, pp. 1823-1827, 2000.

[18] A. R. Shah, D. Gozal, and T. G. Keens, "Determinants of aerobic and anaerobic exercise performance in cystic fibrosis," American Journal of Respiratory and Critical Care Medicine, vol. 157, no. 4, pp. 1145-1150, 1998.

[19] G. P. Boucher, L. C. Lands, J. A. Hay, and L. Hornby, "Activity levels and the relationship to lung function and nutritional status in children with cystic fibrosis," American Journal of Physical Medicine \& Rehabilitation, vol. 76, no. 4, pp. 311-315, 1997.

[20] H. Hebestreit, S. Kieser, S. Rudiger et al., "Physical activity is independently related to aerobic capacity in cystic fibrosis," European Respiratory Journal, vol. 28, no. 4, pp. 734-739, 2006. 


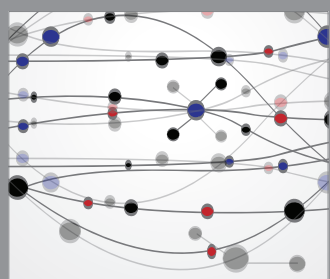

The Scientific World Journal
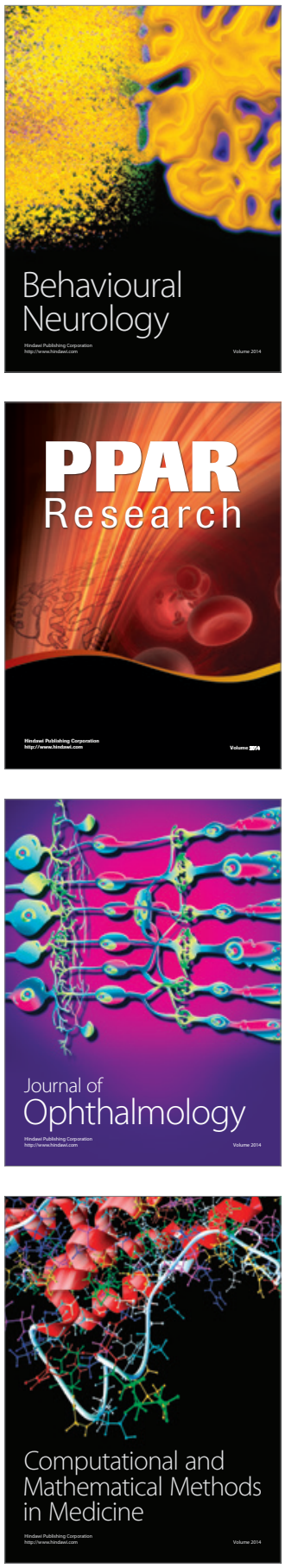

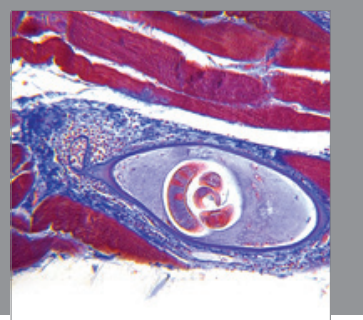

Gastroenterology

Research and Practice
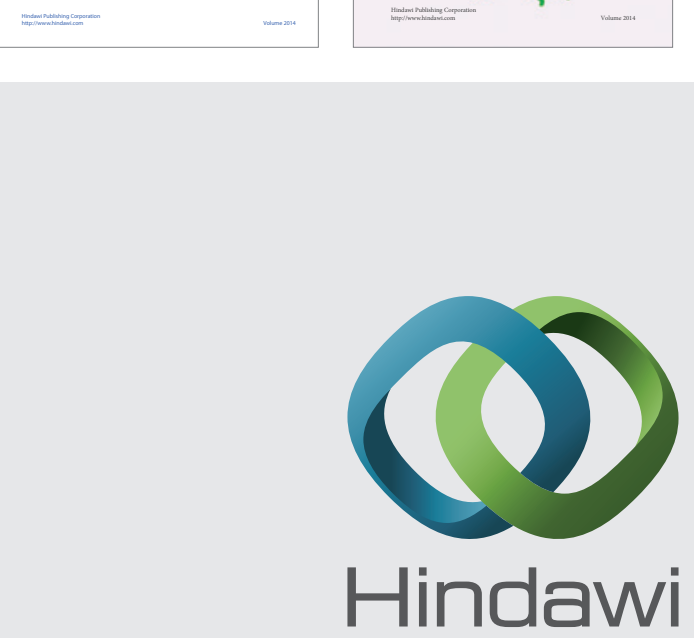

Submit your manuscripts at

http://www.hindawi.com
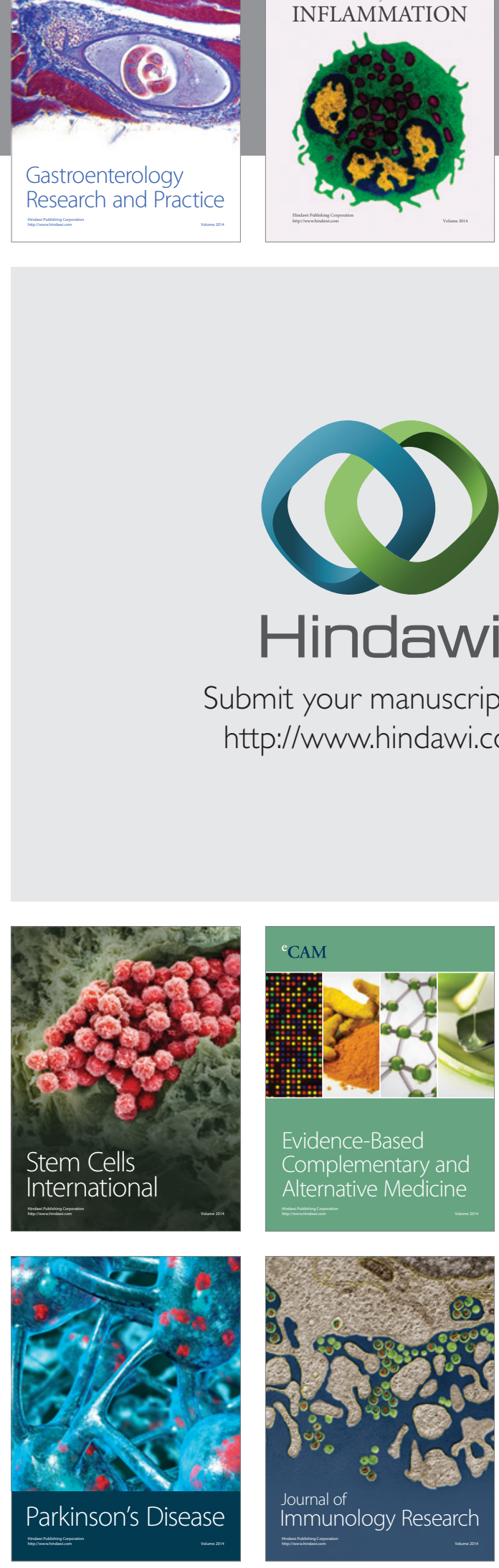

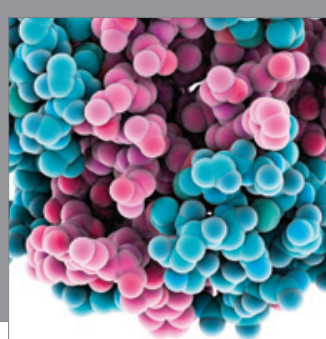

Diabetes Research
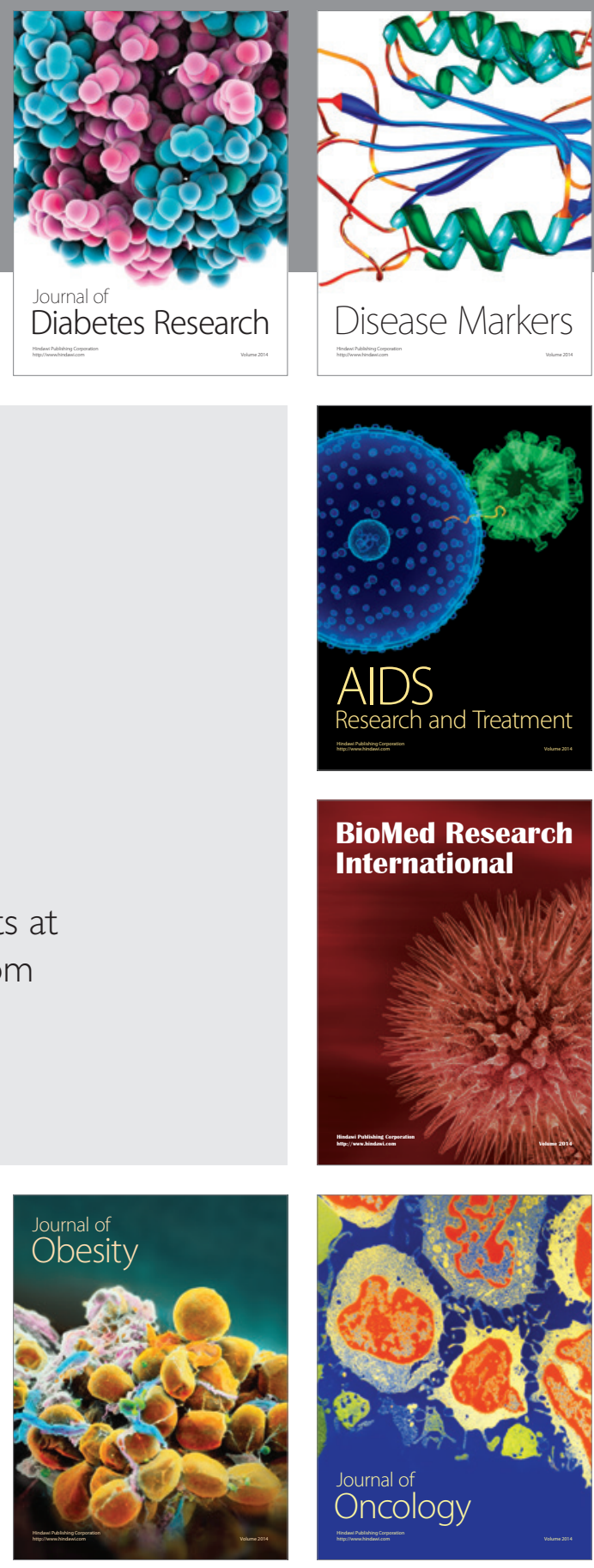

Disease Markers

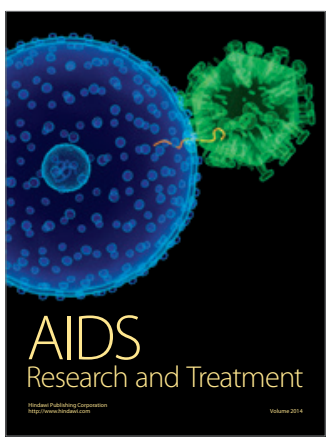

BioMed Research

International
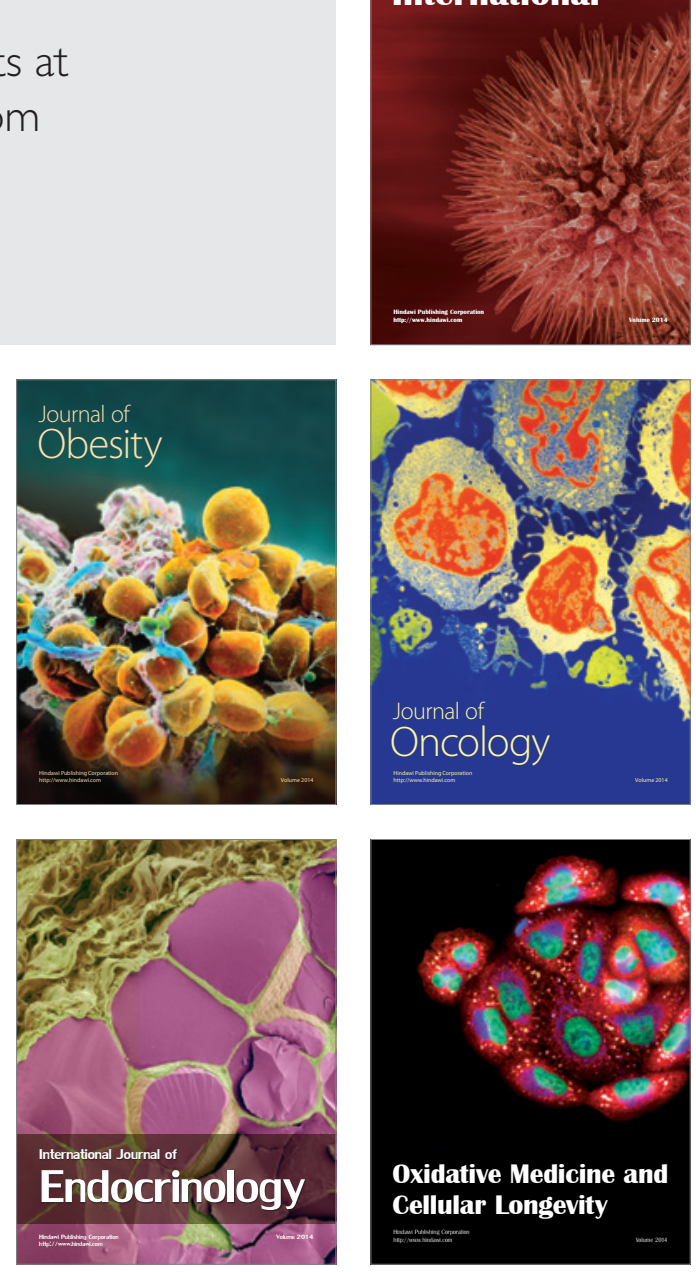